\title{
The Molecular Disk in the Cloverleaf Quasar
}

\author{
S. Venturini \\ Dept. of Physics \& Astronomy, State University of New York, Stony Brook, \\ NY 11794-3800 \\ Stefano.Venturini@sunysb.edu \\ P. M. Solomon \\ Astronomy Program, State University of New York, Stony Brook, NY 11794 \\ PSOLOMON@sbastk. ess. sunysb.edu
}

\begin{abstract}
We propose a new interpretation for the $\mathrm{CO}$ emitting region of the Cloverleaf (H1413+1143), a gravitationally lensed QSO. We proceed by fitting a two-galaxy lensing model directly to the IRAM $\mathrm{CO}(7-6)$ data rather than to the optical HST image. From the fit we infer that the $\mathrm{CO}$ source might be a disk-like structure with a characteristic radius $R \geq 790 \mathrm{pc}^{1}$, a value similar to that of the $\mathrm{CO}$ emitting regions present in nearby starburst ultraluminous infrared galaxies.
\end{abstract}

Subject headings: Gravitational lensing — quasars: individual (H1413+1143, Cloverleaf) — radio lines:galaxies

\section{Introduction}

$\mathrm{H} 1413+1143$, also known as the Cloverleaf, is a four bright image component (labeled from A to D as in Figure 1) broad absorption line QSO at a redshift of $z=2.55$ first found by Hazard et al. (1984) and subsequently identified as a lensed object by Magain et al. (1988).

The earliest indication that molecular emission lines could be present in the Cloverleaf spectrum came from the strong submillimeter dust continuum detected by Barvainis, Antonucci \& Coleman (1992) using the James Clerk Maxwell Telescope. Indeed, the CO(3-2)

\footnotetext{
${ }^{1}$ In the currently widely accepted cosmology: cosmological constant $\Omega_{\Lambda}=0.7$, matter content $\Omega_{m}=0.3$, and Hubble constant $H_{0}=65 \mathrm{~km} \mathrm{~s}^{-1} \mathrm{mpc}^{-1}$.
} 
line emission was subsequently found by Barvainis et al. (1994) using the Instituto de Radioastronomia Milimétrica (IRAM) $30 \mathrm{~m}$ telescope. This detection was soon followed by the detection of multiple CO transitions lines by Barvainis et al. (1997). An interferometric map of the $\mathrm{CO}(3-2)$ emission was obtained by Wilner, Zhao \& Ho (1995) using the BerkeleyIllinois-Maryland Array, but with a too low spatial resolution to resolve the CO gas structure. The first interferometric map of the Cloverleaf in which the $\mathrm{CO}(7-6)$ emission was partially resolved was obtained by Yun et al. (1997) using the Owens Valley Millimeter Array (MMA). A second map with sub-arcsecond resolution of the $\mathrm{CO}(7-6)$ line was obtained by Alloin et al. (1997) using the Institut de Radioastronomie Millimétrique (IRAM) interferometer and was further enhanced by Kneib et al. (1998a) with new subsequent observations. The enhanced $\mathrm{CO}(7-6)$ map fully resolves the four different components of the Cloverleaf.

The first formulation of lensing models by Kayser et al. (1990) used the optical images obtained with the Danish $1.54 \mathrm{~m}$ telescope at the European Southern Observatory and the Very Large Array (VLA) observations at the National Radio Astronomy Observatory. The lensing mass distribution of the two proposed models were described respectively by a singular isothermal elliptical galaxy and two singular isothermal spherical galaxies of equal masses. The model was constrained using the optical data while the VLA image was explained by adding radio features to the lensed source. The resulting fit also suggested the hypothesis that microlensing effects might be important.

Yun et al. (1997) used the Hubble Space Telescope (HST) images made available by Falco (1993) to constrain the lensing geometry. The lens was modeled as an elliptical potential with an external shear. The CO source was subsequently constrained using the derived lensing model and compared to the MMA CO(7-6) data. Yun et al. (1997) also found that the magnification ratios of Keck K-band images obtained from M. Pahre (1996, private communication to Yun et al. 1997) reproduced the HST magnification ratios. It was interpreted as an indication of microlensing being unimportant. However, Østensen et al. (1997) found evidence that the $\mathrm{D}$ component of the lensed image is affected by microlensing.

Kneib et al. (1998a) also used the HST images to constrain the lensing geometry. The two lensing mass distributions were described respectively by two truncated elliptical mass distributions (galaxy + dark halo) and a truncated elliptical mass distribution with an external shear (galaxy + cluster). The enhanced IRAM interferometric CO(7-6) map was then used to constrain the size of the CO source. For these two models, the estimated characteristic radii are respectively 300 pc and 100 pc.

In a subsequent observation, Kneib, Alloin \& Pelló (1998b) detected a possible candidate for the lensing galaxy on HST images. Chae \& Turnshek (1999) used the HST images and the position of the detected galaxy to constrain the lensing model. The lensing galaxy 
mass distribution was modeled by elliptical generalized power-law distributions as in Chae, Khersonsky \& Turnshek (1998). The proposed models used one single galaxy, one galaxy with external shear, and two galaxies as lenses. The two galaxies model gave the best fit and also the highest microlensing probability to the D component. Chae et al. (2001) observed polarization and intensity variations on HST optical images and suggested that microlensing effects are indeed present on the D component of the lensed image.

In this paper we use the improved IRAM CO(7-6) map by Kneib et al. (1998a) to constrain a two-galaxy lensing model. The mass distribution of each galaxy is described by an elliptical generalized power-law distribution as in Chae et al. (1998). We are thus able to derive an intrinsic size for the molecular source and to compare it to the size of nuclear disks present in local ultraluminous infrared galaxies (ULIRGs ${ }^{2}$ ). We actually find that the $\mathrm{CO}$ source has a characteristic radius of $790 \mathrm{pc}$. We also compare our constrained lensing model geometry to lensing geometries present in literature.

This paper is organized in the following way: Section 2 describes the data we use, Section 3 describes the model and the fitting procedures adopted, Section 4 presents our main results, Section 5 provides discussion, and in Section 6 we state our concluding remarks.

\section{The Data}

In our fitting procedure we use the velocity-integrated version of the improved IRAM CO(7-6) map by Kneib et al. (1998a). This map has been kindly provided to us by R. Barvainis.

\section{The Model}

We make two main assumptions in modeling the IRAM $\mathrm{CO}(7-6)$ data. First, we make the assumption that extinction along the line of sight is negligible. Second, we assume that microlensing is negligible in determining the overall geometry of the lensing system. These two assumptions together allow us to compare the map produced by our model to the IRAM $\mathrm{CO}(7-6)$ map.

\footnotetext{
${ }^{2}$ ULIRGs have by definition high $8-1000 \mu$ m luminosities, i.e. $\mathrm{L}_{\mathrm{IR}}[8-1000 \mu \mathrm{m}] \geq 10^{12} \mathrm{~L}_{\odot}$.
} 


\subsection{The Cosmology}

Angular-diameter distances that relate angular separations to linear scales in the object frame are one of the key quantities that enter the lens equation (Schneider, Ehlers \& Falco 1992):

$$
\boldsymbol{\eta}=\frac{D_{s}}{D_{l}} \boldsymbol{\zeta}-D_{s l} \boldsymbol{\alpha}(\boldsymbol{\zeta})
$$

where $\boldsymbol{\eta}$ is the position vector of the QSO in the source plane and $\boldsymbol{\zeta}$ the impact vector of the emitted light ray on the lens plane, $\boldsymbol{\alpha}$ being the deflection angle. $D_{l}$ and $D_{s}$ are the angulardiameter distances of the lens and the source from the observer respectively, while $D_{s l}$ is the angular-diameter distance of the source from the lens (see Figure 2). These distances are computed by integrating a Dyer-Roeder differential equation (Dyer \& Roeder 1973) for the appropriate cosmology. For the cosmology we use $\left(\Omega_{\Lambda}=0.7, \Omega_{m}=0.3\right)$, the equation is (Kantowski \& Thomas 2001):

$$
\begin{aligned}
(1+z)\left(\Omega_{m}(1+z)^{3}+\Omega_{\Lambda}\right) \frac{d^{2} D}{d z^{2}}+\left(\frac{7}{2} \Omega_{m}(1+z)^{3}\right. & \left.+2 \Omega_{\Lambda}\right) \frac{d D}{d z}+ \\
& +\frac{3}{2} \alpha \Omega_{m}(1+z)^{2} D=0
\end{aligned}
$$

where $D$ is the angular-diameter distance and $\alpha \in[0,1]$ is the so-called smoothness parameter. We do not estimate $\alpha$ but we analyze subsequently its impact on the source linear dimensions (see below). For an observer at $z_{1}$ and an object at $z_{2}$, the differential equation has the following boundary conditions:

$$
\begin{aligned}
D\left(z_{1}\right) & =0 \\
\frac{d D}{d z}\left(z_{1}\right) & =\frac{c}{H_{0}} \frac{\operatorname{sign}\left(z_{2}-z_{1}\right)}{\left(1+z_{1}\right) \sqrt{\Omega_{m}\left(1+z_{1}\right)^{3}+\Omega_{\Lambda}}}
\end{aligned}
$$

where $c$ is the speed of light, $H_{0}=65 \mathrm{~km} \mathrm{~s}^{-1} \mathrm{mpc}^{-1}$ is the Hubble constant and $D(z)$ is the angular-diameter distance of an object at redshift $z$ seen by the observer at redshift $z_{1}$.

\subsection{The Lens}

The model assumes the presence of two lensing galaxies at a redshift of $z=1.55$. Each galaxy is modeled as an elliptical generalized power-law mass distribution. The resulting potential responsible of the lensing can be computed using the equations derived in Chae et al. (1998). The redshift of the lensing object candidate found by Kneib et al. (1998b) is not known and the assumed value of $z=1.55$ is consistent with the redshift of observed 
absorbers in the line of sight (Magain et al. 1988; Turnshek et al. 1988). Nevertheless, the angular-diameter distances to the lens and from the lens to the QSO depend on the redshift of the lensing object as seen previously. But, since these distances affect only the linear size of the lensing galaxy and the critical density of the lens, the inferred properties of the $\mathrm{CO}$ source are not affected by the assumption made.

The elliptical generalized power-law mass distribution takes the following form (Chae et al. 1998):

$$
k(\boldsymbol{x})=k(r, \phi)=k_{0}\left\{1+\left(\frac{r}{r_{0}}\right)^{2}\left[1+e \cos 2\left(\phi-\phi_{0}\right)\right]\right\}^{-\mu-1},
$$

where $k_{0}$ is the mass surface density at $r=0$ (i.e. the center of the distribution) in units of the critical mass density (defined as $c^{2} D_{s} / 4 \pi G D_{l} D_{s l}$, where $G$ is the gravitational constant), $e$ is the eccentricity, $\phi_{0}$ is the standard position angle, $r_{0}$ is the core radius and $\mu$ the radial index ( $\mu=-1 / 2$ for an isothermal distribution). To these 5 free parameters, we have to add 2 more that describe the position of the center of the distribution on the lens plane. The total number of free parameters that describe the two galaxies distribution is therefore 14 . The only parameter that we do not minimize is the core radius. We arbitrarily keep it at a fixed value of $1.76 \times 10^{-4}$ arcsec. ( $1.7 \mathrm{pc}$ at the redshift of $\left.z=1.55\right)$ for both galaxies, a value similar to the one in Chae \& Turnshek (1999). This is primarily due to the behavior of the mass density distribution for rays that are far from the core:

$$
k(r, \phi) \approx k_{0} r_{0}^{2 \mu+2}\left\{r^{2}\left[1+e \cos 2\left(\phi-\phi_{0}\right)\right]\right\}^{-\mu-1} .
$$

Therefore, a change in the core radius $r_{0}$ can be very efficiently compensated by a change in the density $k_{0}$, leading to a flat (actually almost flat) direction in parameter space, a deadly trap for a minimization routine. The smallness of the value of the core radius is dictated by the need of a rapid convergence in the numerical evaluation of the series expansion of the deflection angle given in Chae et al. (1998).

The source is simply being modeled as a two dimensional Gaussian surface brightness distribution:

$$
I(x, y)=I_{0} \exp \left\{-\frac{x^{2}}{2 \sigma_{x}^{2}}-\frac{y^{2}}{2 \sigma_{y}^{2}}\right\},
$$

where $I_{0}$ is the central brightness, $\Delta x=\sqrt{2 \ln 2} \sigma_{x}$ and $\Delta y=\sqrt{2 \ln 2} \sigma_{y}$ are the half width at half maximum (HWHM) of the Gaussian while $x$ and $y$ are the coordinates in a coordinate system rotated by a standard position angle $\psi_{0}$. Two more free parameters describe the position of the center of the brightness distribution on the source plane. The total number of free parameters for our model is then 17, having kept the radial index of both galaxies equal. 


\subsection{The Fitting Procedure}

To constrain the parameters of our model, we proceed by minimizing the following $\chi_{\text {d.o.f. }}^{2}$ defined as:

$$
\chi_{\text {d.o.f. }}^{2}=\frac{\sum_{\text {pix }}\left[I_{\text {data }}(\text { pix })-I_{\text {model }}(\text { pix })\right]^{2} / \sigma^{2}}{N_{\text {pix }}-N_{\text {par }}},
$$

where the sum is carried over the pixels of the image. In our case the image is an array of $28 \times 28$ pixels, for a total of $N_{\text {pix }}=784$. We obviously have $N_{\text {par }}=17 . I_{\text {data }}($ pix $)$ and $I_{\text {mod }}($ pix $)$ are the intensity in the data and in the model maps of corresponding pixels. $\sigma$ is the noise level of the IRAM CO(7-6) map (Alloin et al. 1997). We determine the values of these 17 parameters by minimizing such $\chi_{\text {d.o.f }}^{2}$. Due to the large amount of parameters, the choice of a minimization procedure fell on a simulated annealing algorithm such as the one described in Teukolsky, Vetterling \& Flannery (1992). As a remark, the number of data

points included in the definition of the $\chi_{\text {d.o.f. }}^{2}$ is carefully chosen in order not to make our model over-parametrized but also to have a high signal to noise ratio on the overall image. In order to do so, the original map has been trimmed down to the smallest subset, shown in Figure 1, containing all the CO emission from the Cloverleaf.

\section{Results}

\subsection{Our Results}

From Figure 3, we can see that the model reproduces the four images of the lensed QSO. The good agreement with the data is clearly visible in Figure 4 which is the difference between the data image and the image computed using our model. The good agreement is also confirmed by the $\chi_{\text {d.o.f }}^{2}=2.2$. From our model we infer that the emission from the QSO is magnified 11 times. We also notice that the position of one of the lensing galaxies is roughly consistent with the position of the candidate lensing galaxy found by Kneib et al. (1998b). The parameters of the fit that describe the lensing galaxies as well as the parameters that describe the source are listed in Table 1.

\subsection{Comparison with Previous Models}

The first model we compare to is Model 1 from Chae \& Turnshek (1999). The characteristics of the lenses are rather similar as far as the orientation of the mass isocontours are concerned $\left(33^{\circ}\right.$ and $27^{\circ}$ compared to $24^{\circ}$ and $\left.37^{\circ}\right)$. The eccentricities are somewhat different (0.85 and 0.5 compared to 0.39 and 0.6 ). The relative position of the two lensing galaxies 
are also similar, separated by a distance of 0.36 arcseconds instead of 0.5 and with a position angle of $146^{\circ}$ instead of $130^{\circ}$. However there is a big difference in the mass distribution since in our model the two galaxies have similar masses while the galaxies in Model 1 have a mass ratio of about 1:5.

The subsequent models we consider are from Kneib et al. (1998a). These models differ substantially from our model since we use two galaxies as lens while Kneib et al. (1998a) use a single galaxy, and a galaxy with an external shear. The marked difference occurs in the size of the CO source. We obtain a HWHM of $790 \times 680 \mathrm{pc}$, a value much larger than the values of $300 \times 150 \mathrm{pc}$ and $100 \times 70 \mathrm{pc}$ found by Kneib et al. (1998a). The magnification of 11 that we find is also smaller than the magnification found by Kneib et al. (1998a) that is roughly between 18 and 30 .

\section{Discussion}

By fitting our model directly the IRAM $\mathrm{CO}(7-6)$ data, we show that it is indeed possible to constrain a lensing geometry even without the use of high resolution optical data such as HST images. Moreover, the rather good agreement between our model and the one derived by Chae \& Turnshek (1999), using optical HST images and a totally different fitting procedure, can be taken as an indication of the robustness of the 2 galaxies model. Since the derived position of one of the lensing galaxies seems to agree with the position of the candidate lensing object observed by Kneib et al. (1998b), there is an indication that one of the lensing objects has indeed been found.

The smoothness parameter $\alpha$ measures the fraction of the mass smoothly distributed in the universe and not clumped into objects. We do not try to evaluate this parameter present in the Dyer-Roeder equation, but we analyze the behavior of the angular-diameter distance as it varies. The angular-diameter distance for the redshift of the Cloverleaf $z=2.55$ does not vary more than $26 \%$ as $\alpha$ ranges from 0 to 1 . We therefore fix its value to 0.5 , hence making less than a $13 \%$ error in the linear dimensions of the $\mathrm{CO}$ source.

The effective size $(\mathrm{HWHM}) 0.08^{\prime \prime} \times 0.07^{\prime \prime}$ of the brightness profile translates into a size of $790 \times 680 \mathrm{pc}$ at the redshift $z=2.55$ of the Cloverleaf for the adopted cosmology. We take this as an indication that the source might be a disk-like structure with a HWHM radius of $790 \mathrm{pc}$ inclined by roughly $30^{\circ}$ from face on. We list its intrinsic properties in Table 2 where we show the derived CO luminosity (Solomon, Downes \& Radford 1992). In Table 3 we compare the size and CO luminosity of the source for different cosmologies. It is worth noting that the size of the source is indeed similar to the size of nuclear molecular 
disks present in nearby ULIRGs (Downes \& Solomon 1998) as can be seen in Table 4. Even without the full improved IRAM CO(7-6) map available, a rough evaluation of the dynamical mass can be made by assuming that the linewidth of the $\mathrm{CO}(7-6)$ is due to the rotation of the disk:

$$
M_{d y n} \approx \frac{\Delta V^{2} R}{G}=5.1 \times 10^{10} \mathrm{M}_{\odot},
$$

where $\Delta V$ is the FWHM of $376 \mathrm{~km} \mathrm{~s}^{-1}$ found by Alloin et al. (1997) corrected for the assumed inclination of $30^{\circ}, R$ is the HWHM radius of $790 \mathrm{pc}$ and $G$ is the gravitational constant. Recalling that for filled disks the CO luminosity traces the geometric mean of the dynamical mass and the gas mass (Downes, Solomon \& Radford 1993), we obtain:

$$
M_{\text {gas }}=\alpha^{2} 1.8 \times 10^{10} \mathrm{M}_{\odot}
$$

where $\alpha$ is the conversion factor between $M_{\text {gas }}$ and $L_{C O}^{\prime}$. Since the gas mass cannot exceed the dynamical mass, we have the following constrain: $\alpha \leq 1.7$. This value of $\alpha$ is almost 3 times lower that the value of 4.8 found for our Galaxy (Solomon \& Barrett 1991), but it is consistent with the value of about 0.8 found by Downes \& Solomon (1998) for nearby ULIRGs.

We can also have a rough estimate of the size of the Cloverleaf by considering it a black body, made of optically thick dust. We can estimate the temperature of the dust using the $350 \mu \mathrm{m}$ (roughly $100 \mu \mathrm{m}$ in the rest frame) and $100 \mu \mathrm{m}$ (roughly $30 \mu \mathrm{m}$ in the rest frame) flux ratio (see Andreani, Franceschini \& Granato 1999, and references therein):

$$
\frac{F_{100} m_{350}}{F_{350} m_{100}}=\frac{\lambda_{350}^{3}}{\lambda_{100}^{3}} \frac{\exp \left(h c(z+1) / \lambda_{350} k T\right)-1}{\exp \left(h c(z+1) / \lambda_{100} k T\right)-1},
$$

where $F_{\lambda}$ (with $\lambda$ in $\mu m$ ) are the observed fluxes at the observed $\lambda$ wavelength, $m_{\lambda}$ the flux magnification, $z$ the redshift of the Cloverleaf and $T$ the black body temperature. We find that the black body temperature is roughly $135 \mathrm{~K}$, having assumed equal magnification for both fluxes. We estimate a bolometric luminosity of roughly $1.1 \times 10^{14} \mathrm{~L}_{\odot}$ from the spectral energy distribution (Granato et al. 1996), assuming an average magnification of 11 . This leads to an estimate for the black body size of the dust region:

$$
R_{b b}=R_{\odot}\left(\frac{L}{L_{\odot}}\right)^{1 / 2}\left(\frac{T_{\odot}}{T}\right)^{2}
$$

where $L_{\odot}, R_{\odot}, T_{\odot}$ are the luminosity, radius and black body temperature of the sun. We find $R_{b b} \simeq 430 \mathrm{pc}$. Since the true size must be greater than the optically thick size of $430 \mathrm{pc}$, the value of $910 \mathrm{pc}$ found by Granato et al. (1996) and our value of $790 \mathrm{pc}$ for the $\mathrm{CO}(7-6)$ emitting region are acceptable while the estimates of Kneib et al. (1998a) of 300 pc and 100 pc are much too small. 
As a last remark, we would like to stress that we have been comparing different lines: the $\mathrm{CO}(7-6)$ one of the Cloverleaf and the $\mathrm{CO}(1-0)$ one of nearby ULIRGs. It is indeed true that gas conditions and sizes of the emitting regions that these two lines probe are

different. Nevertheless, it seems reasonable to assume that the size of the $\mathrm{CO}(7-6)$ emitting region gives a lower bound to the size of the $\mathrm{CO}(1-0)$ emitting region. It is more difficult though to extend such simple considerations to the brightness temperature, given its complex dependence on the local gas conditions, and therefore to the CO luminosity. Unfortunately, with only the $\mathrm{CO}(7-6)$ high resolution interferometric data available, it is not possible to further investigate the observed similarities between these objects.

\section{Conclusions}

1. Size of the $C O(7-6)$ emitting region: The size of the CO source has a lower bound given by the size of the $\mathrm{CO}(7-6)$ line emitting region. The characteristic size (HWHM) of the $\mathrm{CO}(7-6)$ line emitting region varies roughly from 410 pc to 890 pc depending on the cosmology. These sizes are similar to the size of the nuclear molecular disks present in nearby ULIRGs.

2. Molecular gas content: Assuming that the source is indeed a disk-like structure, we found an upper bound to the molecular gas mass of a few $10^{10} \mathrm{M}_{\odot}$. Assuming a conversion factor of 0.8 between $M_{\text {gas }}$ and $L_{C O}^{\prime}$, we find a molecular gas content of $1.4 \times 10^{10} \mathrm{M}_{\odot}$, consistent with the amount of molecular gas found in nearby ULIRGs.

3. Starburst powered region: The large size of the CO source (about $1 \mathrm{kpc}$ ) seems to rule out a scenario in which the molecular gas is concentrated in a very small region around the central AGN. This is consistent with the molecular gas being heated by a starburst, as in nearby ULIRGs. Therefore, two distinct energy sources could coexist in the Cloverleaf: a central AGN and an extended starburst region. However, the possibility of having the molecular gas heated by a central AGN cannot be completely ruled out (see Granato et al. 1996).

We would like to thank R. Barvainis for having made the improved IRAM CO(7-6) integrated map available to us. We would also like to thank A. Evans and J. S. Kim for helpful discussions. 


\section{REFERENCES}

Alloin, D., Guilloteau, S., Barvainis, R., Antonucci, R. \& Tacconi, L. 1997, A\&A, 321, 24

Andreani, P., Franceschini, A. \& Granato, G. 1999, MNRAS, 306, 161

Barvainis, R., Antonucci, R. \& Coleman, P. 1992, ApJ, 399, L19

Barvainis, R., Tacconi, L., Antonucci, R., Alloin, D. \& Coleman, P. 1994, Nature, 371, 586

Barvainis, R., Maloney, P., Antonucci, R. \& Alloin, D. 1997, ApJ, 484, 695B

Chae, K.-H., Khersonsky, V. K. \& Turnshek, D. A. 1998, ApJ, 506, 80

Chae, K.-H. \& Turnshek, D. A. 1999, ApJ, 514, 587

Chae, K.-H., Turnshek, D. A., Schulte-Ladbeck, R. E., Rao, S. M. \& Lupie, O. L. 2001, ApJ, 561,653

Downes, D., Solomon, P. M. \& Radford, S. J. E 1993, ApJ, 414, L13

Downes, D. \& Solomon, P. M. 1998, ApJ, 507, 615

Downes, D. \& Solomon, P. M. 2002, ApJ, in press (ApJ preprint doi:10.1086/344594)

Dyer, C. C. \& Roeder, R. C. 1973, ApJ, 180, L13

Falco E. E. 1993, in Gravitational Lenses in the Universe, ed. J. Surdej et al. (Université de Liège), 127

Granato, G. L., Danese, L. \& Franceschini, A. 1996, ApJ, 460, L11

Hazard, C., Morton, D. C., Terlevich, R., \& McMahon, R. 1984, ApJ, 282, 33

Kantowski, R. \& Thomas, R. C. 2001, ApJ, 561, 491

Kayser, R., Surdej, J., Condon, J., Kellerman, K., Magain, P., Remy, M. \& Smette, A. 1990, ApJ, 384, 1

Kneib, J.-P., Alloin, D., Mellier, Y., Guilloteau, S., Barvainis, R. \& Antonucci, R. 1998 (a), A\&A, 329, 827

Kneib, J.-P., Alloin, D., \& Pelló, R. 1998 (b), A\&A, 339, L65

Magain, P., Surdej, J., Swings, J.-P., Borgeest, U., Kayser, R., Kuhr, H., Refsdal, S. \& Remy, M. 1988, Nature, 334, 325 
Østensen, R., et al. 1997, A\&AS, 126, 393

Schneider, P., Ehlers, J. \& Falco, E. E. 1992, Gravitational Lenses (1st ed.; Springer)

Solomon, P. M. \& Barrett, J. W. 1991, in "Dynamics of Galaxies and Their Molecular Cloud Distributions", ed. F. Combes \& F. Casoli (Kluwer Academic Publishers), 235

Solomon, P. M., Downes, D. \& Radford, S. J. E. 1992, ApJ, 398, L29

Teukolsky, S. A., Vetterling, W. T. \& Flannery, B. P. 1992, Numerical Recipes in Fortran, (2d ed.; Cambridge University Press)

Turnshek, D. A., Foltz, C. B., Grillmair, C. J. \& Weyman, R. J. 1988, ApJ, 325, 651

Wilner, D. J., Zhao, J.-H. \& Ho, P. T. P. 1995, ApJ, 452, L91

Yun, M., Scoville, N. Z., Carrasco, J. J. \& Blandford, R. D. 1997, ApJ, 479, L9 




Fig. 1. - Integrated IRAM $\mathrm{CO}(7-6)$ map. The beam is $0.77^{\prime \prime} \times 0.44^{\prime \prime}$ at a P.A. of $15^{\circ}$. Contours are roughly $0.6 \mathrm{Jy} \mathrm{km} \mathrm{s}^{-1}$, i.e. circ. $1 \sigma$. 


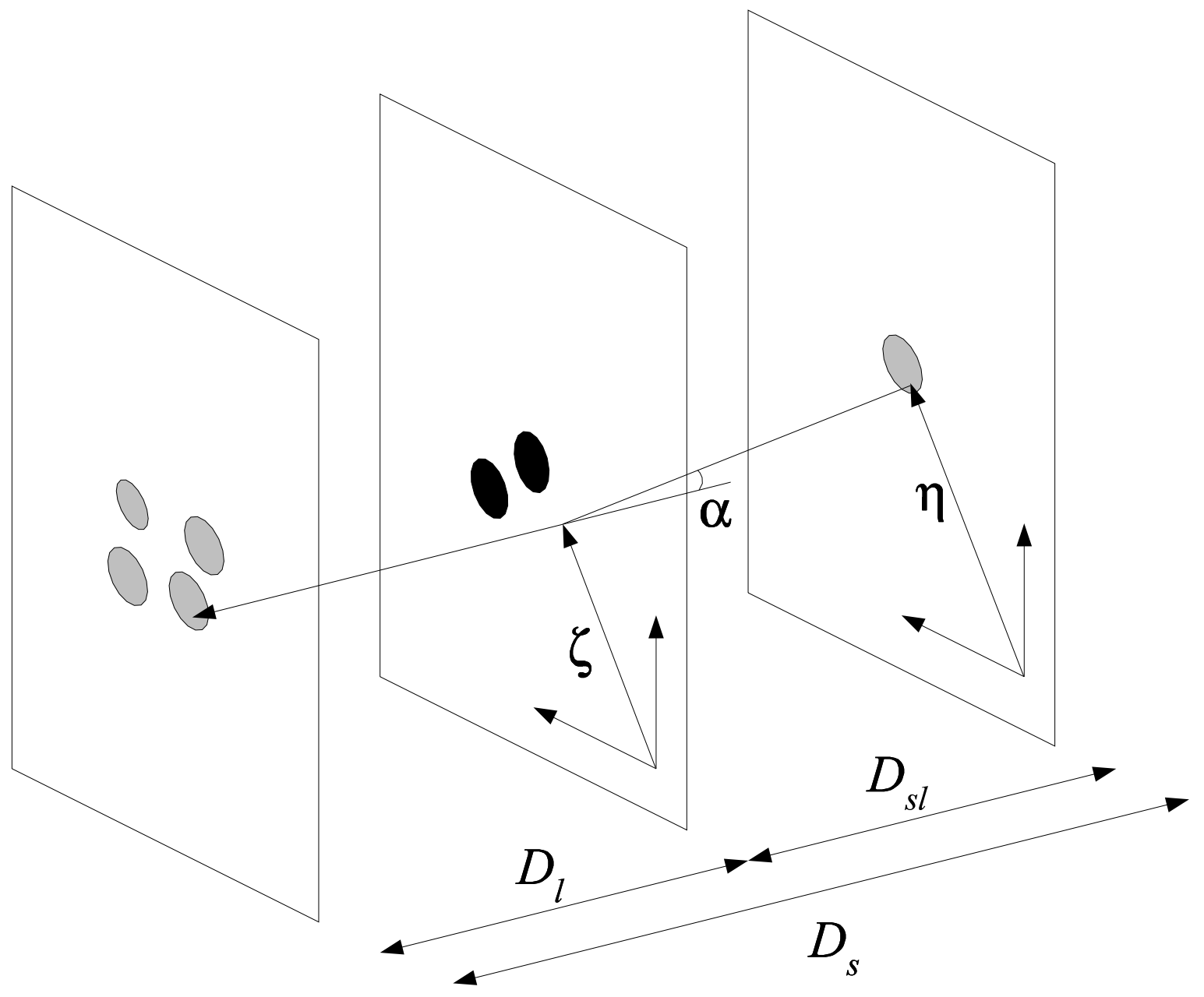

Fig. 2.- A light ray from the source with position vector $\boldsymbol{\eta}$ on the source plane and impact parameter $\boldsymbol{\zeta}$ on the lens plane is deflected by the deflection angle $\boldsymbol{\alpha}$. Also shown are the angular-diameter distances. 


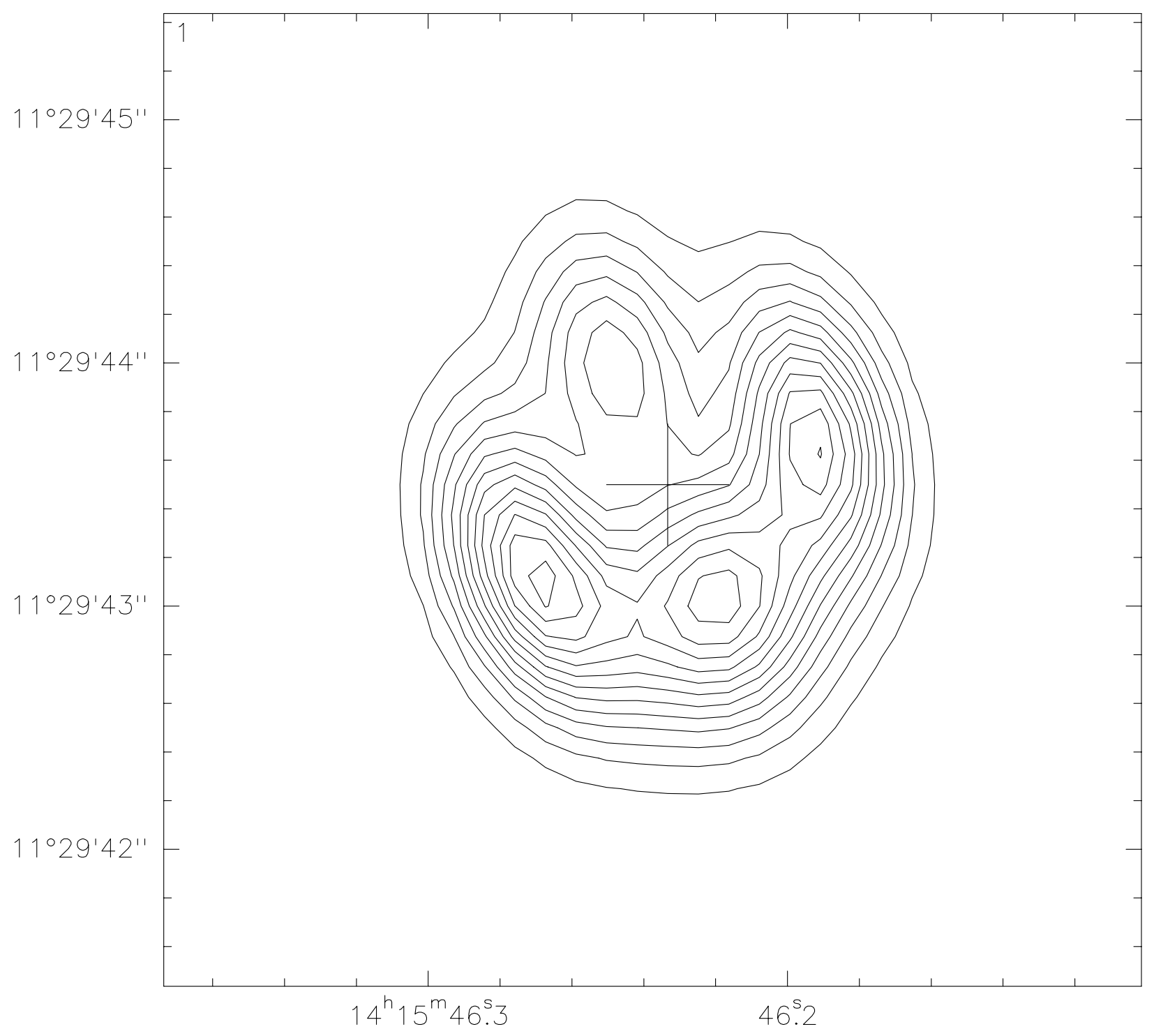

Fig. 3.- Map from the model. The beam is $0.77^{\prime \prime} \times 0.44^{\prime \prime}$ at a P.A. of $15^{\circ}$. Contours are roughly $0.6 \mathrm{Jy} \mathrm{km} \mathrm{s}^{-1}$. 


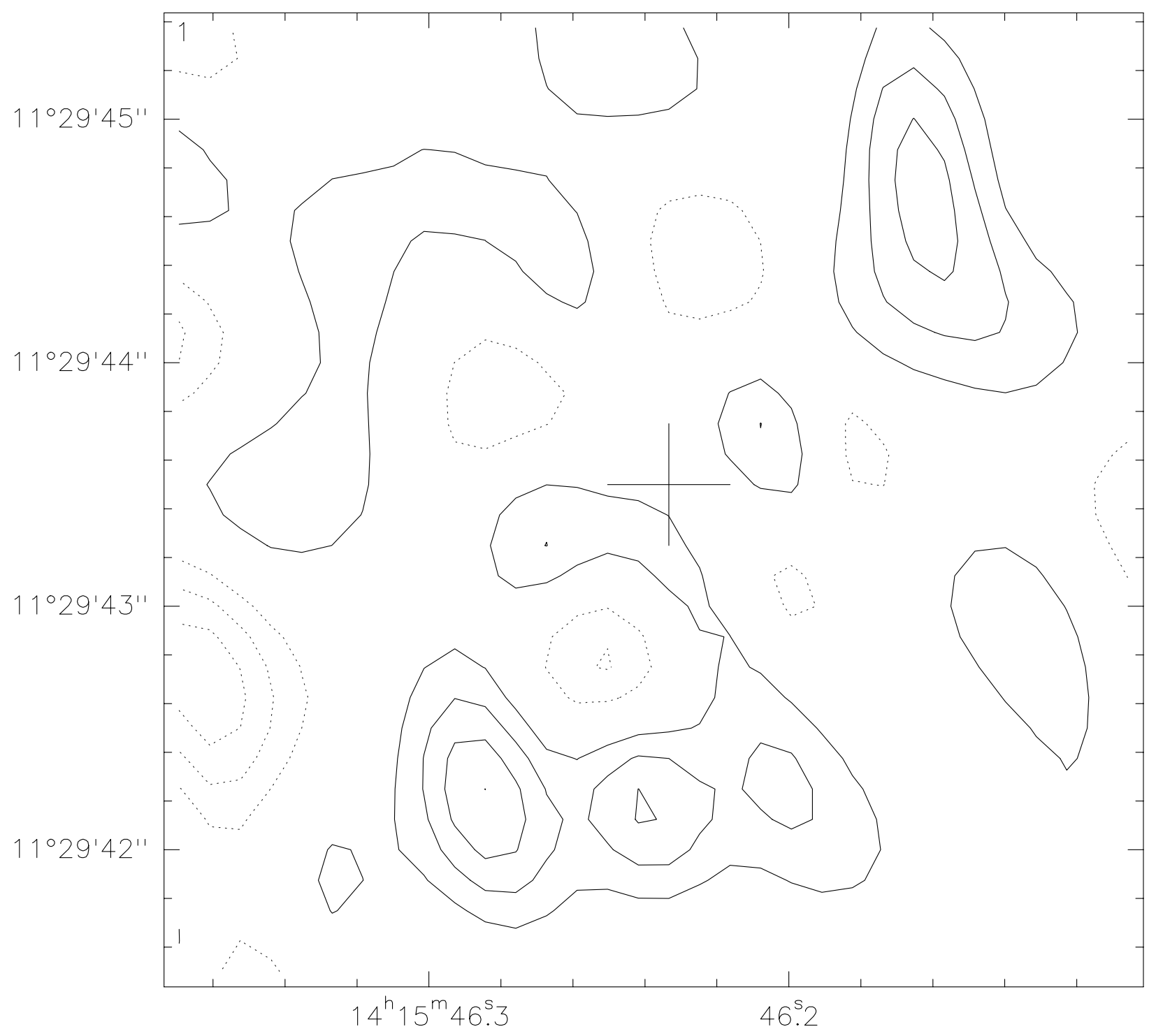

Fig. 4. - Data minus the model. The beam is $0.77^{\prime \prime} \times 0.44^{\prime \prime}$ at a P.A. of $15^{\circ}$. Contours are roughly $0.6 \mathrm{Jy} \mathrm{km} \mathrm{s}^{-1}$, i.e. circ. $1 \sigma$. 
Table 1. Model Parameters ${ }^{\text {a }}$

Lens Parameters

Galaxy 1

$\begin{array}{lc}k_{0}\left(10^{7} \mathrm{M}_{\odot} \mathrm{pc}^{-2}\right) & 1.8 \pm 0.3 \\ e & 0.5 \pm 0.1 \\ \text { P.A. }^{\mathrm{b}} \text { (deg.) } & 27 \pm 9 \\ x_{G}, y_{G}{ }^{\mathrm{c}} \text { (arcsec.) } & 0.21 \pm 0.04,-0.15 \pm 0.05 \\ \mu^{\mathrm{d}} & -0.38 \pm 0.01\end{array}$

Galaxy 2

$\begin{array}{lc}k_{0}\left(10^{7} \mathrm{M}_{\odot} \mathrm{pc}^{-2}\right) & 1.3 \pm 0.2 \\ e & 0.85 \pm 0.05 \\ \text { P.A. }^{\mathrm{b}} \text { (deg.) } & 33 \pm 9 \\ x_{G}, y_{G}{ }^{\mathrm{c}} \text { (arcsec.) } & 0.007 \pm 0.008,0.15 \pm 0.06 \\ \mu^{\mathrm{d}} & -0.38 \pm 0.01\end{array}$

Source Parameters

\begin{tabular}{lc}
\hline$I_{0}\left(10^{4} \mathrm{~K} \mathrm{~km} \mathrm{~s}^{-1}\right)$ & $1.2 \pm 0.3$ \\
$\Delta x, \Delta y$ (arcsec.) & $0.08 \pm 0.01,0.07 \pm 0.01$ \\
P.A. ${ }^{\mathrm{b}}$ (deg.) & $55 \pm 13$ \\
$x_{S}, y_{S}{ }^{\mathrm{c}}$ (arcsec.) & $0.08 \pm 0.01,-0.05 \pm 0.01$ \\
\hline
\end{tabular}

${ }^{a}$ Error is computed as $\chi_{\text {d.o.f.min }}+1.0$.

${ }^{b}$ Position angle is East of North.

${ }^{\mathrm{C}}$ Offsets are with respect to the center of the IRAM CO(7-6) map at $14^{\mathrm{h}} 15^{\mathrm{m}} 46^{\mathrm{s}} .233 \mathrm{RA}$ and $11^{\circ} 29^{\prime} 43^{\prime \prime} .50$ DEC, 2000 epoch.

dThe radial index is kept equal for both galaxies.

${ }^{\mathrm{e}}$ Half width at half power. In the adopted cosmology the corresponding linear sizes are (790土 $80,680 \pm 80)$ pc. 
Table 2. Source Properties

\begin{tabular}{lcc}
\hline \hline & $\begin{array}{c}L_{C O}^{\prime} \\
10^{10} \mathrm{~K} \mathrm{~km} \mathrm{~s}^{-1} \mathrm{pc}^{2}\end{array}$ & $\begin{array}{c}L_{C O} \\
10^{8} \mathrm{~L}_{\odot}\end{array}$ \\
\hline Data & $40 \pm 01$ & $68 \pm 03$ \\
Model Lensed & $31 \pm 11$ & $53 \pm 18$ \\
Model Unlensed & $2.8 \pm 1.0$ & $4.8 \pm 1.6$ \\
\hline
\end{tabular}


Table 3. Source Properties in Different Cosmologies

\begin{tabular}{cccc}
\hline \hline$\alpha$ & $\begin{array}{c}L_{C O}^{\prime} \text { Data } \\
10^{10} \mathrm{~K} \mathrm{~km} \mathrm{~s}^{-1} \mathrm{pc}^{2}\end{array}$ & $\begin{array}{c}L_{C O}^{\prime} \text { Model Unlensed } \\
10^{10} \mathrm{~K} \mathrm{~km} \mathrm{~s}^{-1} \mathrm{pc}^{2}\end{array}$ & $\begin{array}{c}(\Delta x, \Delta y)^{\mathrm{a}} \\
\mathrm{pc}\end{array}$ \\
\hline & & & \\
\hline & \multicolumn{3}{c}{$\Omega_{m}=0.3, \Omega_{\Lambda}=0.7, H_{0}=65 \mathrm{~km} \mathrm{~s}^{-1} \mathrm{mpc}^{-1}$} \\
\hline 0.0 & 51 & 3.6 & $(890,770)$ \\
0.5 & 40 & 2.8 & $(790,680)$ \\
1.0 & 30 & 2.1 & $(690,590)$ \\
\hline & $\Omega_{m}=1.0, \Omega_{\Lambda}=0.0, H_{0}=75 \mathrm{~km} \mathrm{~s}^{-1} \mathrm{mpc}^{-1}$ \\
\hline 0.0 & 22 & 1.6 & $(590,510)$ \\
0.5 & 16 & 1.1 & $(490,430)$ \\
1.0 & 11 & 0.7 & $(410,350)$ \\
\hline
\end{tabular}

${ }^{a}$ Half width at half power (HWHM). 
Table 4. Sizes of CO Emitting Regions

\begin{tabular}{lrrrcc}
\hline \hline & CO Line & $\begin{array}{c}\text { Radius }^{\mathrm{a}} \\
\mathrm{pc}\end{array}$ & $\begin{array}{c}\text { True } T_{b} \\
\mathrm{~K}\end{array}$ & $\begin{array}{c}L_{C O}^{\prime}{ }^{\mathrm{b}} \\
10^{9} \mathrm{~K} \mathrm{~km} \mathrm{~s}^{-1} \mathrm{pc}^{2}\end{array}$ & Reference \\
\hline Cloverleaf & $7-6$ & 790 & 32 & 28.0 & 1 \\
VII Zw 31 & $1-0$ & 1300 & 18 & 12.0 & 2 \\
Arp 220 & $1-0$ & 560 & 29 & 7.9 & 2 \\
Mrk 231 & $1-0$ & 540 & 56 & 7.0 & 2 \\
Mrk 273 & $1-0$ & 460 & 36 & 7.4 & 2 \\
IRAS 10214+4724c & $3-2$ & 540 & 40 & 11.0 & 3 \\
& $6-5$ & 540 & 23 & 6.4 & 3 \\
SMM J14011+0252 & $3-2$ & 410 & 35 & 5.7 & 4 \\
& $7-6$ & 480 & 7 & 1.3 & 4 \\
\hline
\end{tabular}

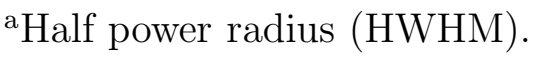

${ }^{\mathrm{b}}$ Intrinsic luminosity.

${ }^{\mathrm{c} A s s u m i n g ~ a ~ m a g n i f i c a t i o n ~ o f ~} 15$.

References. - (1)This article;(2)Downes \& Solomon (1998);(3)Solomon et al. (1992);(4)Downes \& Solomon (2002). 